\title{
An atomistic investigation on the wear of diamond during atomic force microscope tip- based nanomachining of Gallium Arsenide
}

Pengfei Fan ${ }^{1}$, Saurav Goel ${ }^{2,3,4,5}$, Xichun Luo ${ }^{1 *}$, Yongda Yan $^{6}$, Yanquan Geng ${ }^{6}$, Yuzhang

Wang $^{6}$

${ }^{1}$ Centre for Precision Manufacturing, DMEM, University of Strathclyde, UK

${ }^{2}$ School of Engineering, London South Bank University, 103 Borough Road, London SE1 0AA, UK

${ }^{3}$ EPSRC Centre for Doctoral Training in Ultra-Precision Engineering, University of Cambridge and Cranfield University, UK

${ }^{4}$ School of Aerospace, Transport and Manufacturing, Cranfield University, Bedfordshire, MK43 OAL, UK

${ }^{5}$ Department of Mechanical Engineering, Shiv Nadar University, Gautam Budh Nagar, 201314, India

${ }^{6}$ Center for Precision Engineering, Harbin Institute of Technology, Harbin, P. R. China

*Corresponding email: xichun.luo@strath.ac.uk

\section{Abstract}

This paper investigated the wear mechanism of diamond during the atomic force 
microscope (AFM) tip-based nanomachining of Gallium Arsenide (GaAs) using molecular dynamics (MD) simulations. The elastic-plastic deformation at the apex of the diamond tip was observed during the simulations. Meanwhile, a transition of the diamond tip from its initial cubic diamond lattice structure $s p^{3}$ hybridization to graphite lattice structure $s p^{2}$ hybridization was revealed. Graphitization was, therefore, found to be the dominant wear mechanism of the diamond tip during the nanometric cutting of single crystal gallium arsenide for the first time. The various stress states, such as hydrostatic stress, shear stress, and von Mises stress within the diamond tip and the temperature distribution of the diamond tip were also estimated to find out the underlying mechanism of graphitization. The results showed that the cutting heat during nanomachining of GaAs would mainly lead to the graphitization of the diamond tip instead of the high shear stress-induced transformation of the diamond to graphite. The paper also proposed a new approach to quality the graphitization conversion rate of diamond tip.

Keywords: AFM tip-based nanomachining; MD simulation; Diamond tip wear; Single crystal gallium arsenide; Graphitization

\section{Introduction}

Gallium arsenide (GaAs), a high value-added semiconductor material, has unique advantages in contrast to silicon, such as higher electron mobility, wider bandgap and higher temperature resistance [1]-[3]. It has been broadly used in the field of microelectronics and optoelectronics to make smart devices for telecommunication, 
fibre-optic communication, satellite communication, cable television, vehicle navigation system and semiconductor light-emitting diodes [4]-[7]. The elastic modulus and fracture toughness of GaAs is much lower than single crystal silicon [8], which makes it much harder to machine than silicon [9].

AFM tip-based nanomachining of GaAs offers a low-cost solution to perform flexible direct writing [10] with nanometer scale accuracy in a single pass under various conditions, such as ultra-vacuum, liquid and low temperature or high temperature [11]. For example, Hyon et al. [12] employed AFM tip-based nanomachining approach to fabricate patterns as narrow as $10 \mathrm{~nm}$ on the GaAs workpiece. Schumacher et al. [13] obtained a single-electron transistor by fabricating a GaAs/AlGaAs heterostructure surface. Additionally, AFM tip-based nanomachining approach has been successfully applied to generate nanodots, nanolines, two-dimensional (2D), and even threedimensional (3D) nanostructures with serval nanoNewton force [14]-[18]. This is particularly useful for the fabrication of 3D GaAs nanostructures, for instance, the hemisphere cavity for light-emitting diodes quantum devices on the GaAs substrate. Due to the nature of mechanical material removal, AFM tip-based machining outperforms the focussed ion beam (FIB) machining approach [19] in terms of high material removal rate and consistent device performance due to no ion contamination on the fabricated devices.

The diamond succumbs to wear during nanomachining process, leading to the worsening of the machined surface by the virtue of the transition of machining mode from ductile-regime to brittle fracture [20]. Catastrophic wear of diamond tip leads to 
stress concentration in the cutting zone which brings about microcracks and fractures. Correspondingly, an understanding of the wear mechanism of the diamond tip is necessary to mitigate the wear of diamond tip during high-volume production of GaAsenabled devices. This is the motivation of the research paper. Single crystal diamond and polycrystalline diamond tools have been experimentally observed to undergo different wear mechanisms, such as diffusion wear [21], thermo-chemical wear [22], abrasive wear [23], graphitization [24], and chemical diffusion [25]. Nevertheless, experimental technologies are currently limited by the timescales to monitor real-time events such as the wear of material occurring within a few picoseconds where simulations can contribute very strongly as accelerated digital nanomanufacturing tools. Correspondingly, molecular dynamics (MD) simulation has become a favourable choice which is capable of revealing many additional atomistic insights of the tribological phenomena in terms of energy and structural changes [26]. For example, carbon atoms of the diamond tool were observed to transform to graphite structure during single point diamond turning of mold steel by using MD simulation [27]. Goel et al. employed MD simulation to reveal $s p^{3}-s p^{2}$ hybridization order-disorder transformation of carbon atoms when cutting silicon [28] and silicon carbide [29]. Additionally, the diffusion wear of the diamond tool was shown in the study of machining aluminum alloy [30] by utilizing MD simulations. These works have proved that MD simulation is an effective technique to unravel the wear mechanism of diamond cutting tools. However, the wear mechanism of the diamond tool during nanometric cutting of single crystal GaAs has not been revealed yet. Therefore, this paper 
investigates the wear mechanism of the diamond tip using large scale MD simulations. The innovation of this paper is to simulate the atomic force macroscope tip-based nanomachining process on high brittleness single crystal GaAs material to investigate the wear mode of the diamond tip. It is different from the previous simulation works on hard-brittle materials, such as silicon carbide and silicon, in terms of the geometry of the tool top mimicking an AFM tip and the use of the type of potential function. The potential influential factors on the wear of diamond tips, such as thermal effect and high shear stress, were analyzed and discussed in the subsequent sections. Additionally, a quantitative assessment of the graphitization rate of the diamond tip was also proposed.

\section{MD simulation}

\subsection{MD simulation model}

In this study, a 3D MD simulation model for AFM tip-based nanomachining of single crystal GaAs workpiece was established. The simulation was implemented in the opensource code, "Large-scale atomic/molecular massively parallel simulator" LAMMPS [31]. As shown in Fig. 1, a square pyramid diamond tip with the (1 00 ) crystallographic orientation was considered as a non-rigid body with a 1:1 aspect ratio. The tip was used to cut the GaAs on the $(010)$ orientation in the $<100>$ direction. Since the focus of the investigation here is to understand the wear of diamond, the anisotropy aspects of the tool and the workpiece were not reported here. The GaAs workpiece was built with a 
size of $31.0 \mathrm{~nm} \times 10.1 \mathrm{~nm} \times 13.4 \mathrm{~nm}$ in the $\mathrm{X}$ (length), $\mathrm{Y}$ (width), and $\mathrm{Z}$ (height) directions, respectively. The green and blue colour atoms represent the boundary layers and thermostat layers of the tool and workpiece while red and purple colour belongs to gallium and arsenide atoms, respectively, within the GaAs workpiece Newton layers and the yellow atoms represent the Newton atoms of the diamond tool. The thermostat layer was prescribed a Berendsen thermostatic dynamics to dissipate the artificial Joule heat generated during the cutting process.

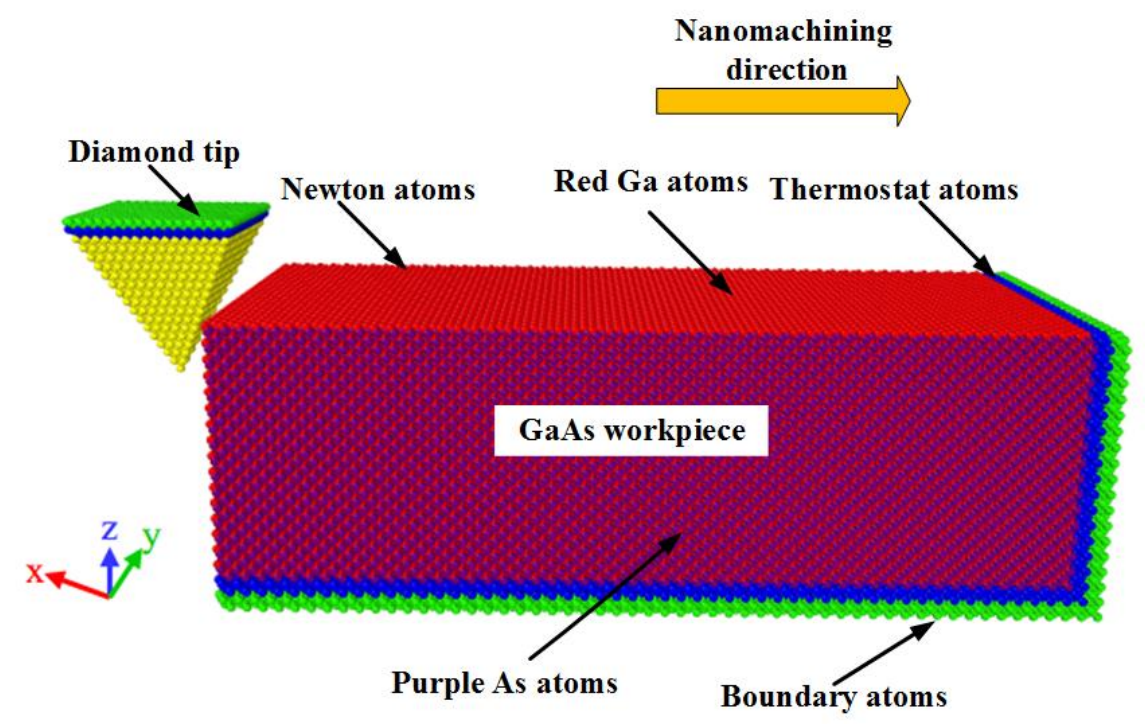

Fig. 1. The MD simulation model for AFM diamond tip-based nanomachining of single crystal GaAs During the simulation, the initial temperature of the GaAs workpiece and diamond tip machining tool were equilibrated at $300 \mathrm{~K}$ by using the Nose-Hoover method [32]. Also, atoms in the $\mathrm{X}, \mathrm{Y}$, and $\mathrm{Z}$ direction were set into shrink-wrapped, shrink-wrapped, and periodic boundary conditions, respectively. The shrink-wrapped boundary condition is capable of wrapping the atoms dropped from the diamond tip while there will be no new atoms at the corresponding position to supplement, which is very suitable to 
investigate the change or transformation of tip structure during nanomachining. Finally, the simulation of cutting experiments was carried out by prescribing a depth of cut of 3 $\mathrm{nm}$ and length of cut of $20 \mathrm{~nm}$ (tip velocity of $200 \mathrm{~m} / \mathrm{s}$ ) under a fixed number of atoms $(\mathrm{N})$, volume $(\mathrm{V})$, and energy $(\mathrm{E})$, so-called NVE microcanonical ensemble. The simulation parameters are shown in detail in Table 1 . The simulation results were visualised and analyzed using visual molecular dynamics (VMD) [33] and Open Visualization Tool (OVITO) software [34].

Table 1

Simulation parameters of MD simulation model

\begin{tabular}{lc}
\hline Substrate dimensions & $31.0 \mathrm{~nm} \times 10.1 \mathrm{~nm} \times 13.4 \mathrm{~nm}$ \\
\hline Number of GaAs substrate atoms & 190080 \\
Machining tool & Diamond tip (wearable) \\
Number of diamond tool atoms & 13107 \\
Crystallographic plane of diamond & $(100)$ \\
tip & \\
GaAs lattice constant & $5.65 \AA$ \\
Diamond lattice constant & $3.57 \AA$ \\
Diamond tip aspect ratio & $1: 1$ \\
Depth of cut & $3 \mathrm{~nm}$ \\
Machining distance & $20 \mathrm{~nm}$ \\
Cutting velocity & $200 \mathrm{~m} / \mathrm{s}$ \\
Crystallographic plane of GaAs & $(010)$ \\
substrate & $300 \mathrm{~K}$ \\
Equilibration temperature & $1 \mathrm{fs}$ \\
Timestep & \\
\hline
\end{tabular}

\subsection{Selection of potential functions}


In this work, the force field consisted of a hybrid scheme such that the Tersoff potential function (C-C interactions), updated Bond-Order Potential (BOP) function (Ga-As interactions) and Ziegler-Biersack-Littmark (ZBL) potential function (for cross interaction between carbon with GaAs atoms) were respectively employed. The classical Tersoff potential function [35] was selected to describe the C-C interactions in the diamond tip machining tool [36]. The calculations of a Tersoff potential function are shown in Eq. (1-8) [37].

$$
\begin{gathered}
E=\sum_{i=j} V_{i j} \\
V_{i j}=f_{C}\left(r_{i j}\right)\left[f_{R}\left(r_{i j}\right)+b_{i j} F_{A}\left(r_{i j}\right)\right] \\
f_{R}\left(r_{i j}\right)=A \exp \left(-\lambda r_{i j}\right) \\
f_{A}\left(r_{i j}\right)=-B \exp \left(-\mu r_{i j}\right) \\
f_{c}\left(r_{i j}\right)=\left\{\begin{array}{l}
1 \\
\frac{1}{2}+\frac{1}{2} \cos \left[\pi \frac{r_{i j}-r}{S-R}\right] \quad R<r_{i j}<S \\
0 \quad r_{i j}>R
\end{array}\right. \\
b_{i j}=\left(1+\beta_{i}^{n} \delta_{i j}^{n}\right)^{-1 / 2 n} \\
\delta_{i j}=\sum_{k \neq i . j} f_{c}\left(r_{i j}\right) g\left(\theta_{i j k}\right) \\
g\left(\theta_{i j k}\right)=\frac{\left(1+c^{2}\right) /\left(d^{2}-c^{2}\right)}{\left[d^{2}+\left(h-\cos \theta_{i j k}\right)^{2}\right]}
\end{gathered}
$$

where $E$ represents the system total energy, the $V_{i j}$ refers to bond energy in the whole atomic bonds, $\mathrm{i}, \mathrm{j}$, and $\mathrm{k}$ mean the atomic label of the system, $f_{C}$ is a smooth cutoff function to restrain the range of the potential, $f_{R}$ is a two-body term, $r_{i j}$ represents the length function of the $\mathrm{i}$ and $\mathrm{j}$ bond, $b_{i j}$ means the bond order term, $F_{A}$ consists of the three-body interactions, $f_{A}$ and $f_{C}$ are the attractive pair potential and smooth cutoff 
function, respectively. $\delta_{i j}$ refers to the atom number in which the atoms $\mathrm{i}$ besides the $\mathrm{ij}$ bond. The $\theta$ stands for the bond angle.

A BOP function based on quantum mechanical theory [38][39] is capable of predicting the structural changes and binding energy trends in the GaAs system, it was adopted to describe the interaction of Ga-Ga, As-As, and Ga-As, which was computed by Eq. (9). The GaAs bond-dependent BOP parameters are shown in Table 2 [40]. The repulsive and bond integral terms were calculated by Goodwin-Skinner-Pettifor (GSP) function [41].

$\mathrm{E}=\frac{1}{2} \sum_{i=1}^{N} \sum_{j=i_{1}}^{i_{N}} \varphi_{i j}\left(r_{i j}\right)-\sum_{i=1}^{N} \sum_{j=i_{1}}^{i_{N}} \beta_{\sigma, i j}\left(r_{i j}\right) \cdot \theta_{\sigma, i j}-\sum_{i=1}^{N} \sum_{j=i_{1}}^{i_{N}} \beta_{\pi, i j}\left(r_{i j}\right) \cdot$

$\theta_{\pi, i j}+U_{\text {prom }}$

where the $r_{i j}$ is the distance function for atoms $i$ and $j, \varphi_{i j}\left(r_{i j}\right)$ is the repulsive energy and short-range two-body function, $\beta_{\sigma, i j}\left(r_{i j}\right)$, and $\beta_{\pi, i j}\left(r_{i j}\right)$ refer to bond integrals, $\theta_{\sigma, i j}$ and $\theta_{\pi, i j}$ are specific bond-orders, $U_{\text {prom }}$ means the promotion energy about spvalent systems. Following the BOP potential function, the cutoff was modified to 11.1 $\AA$ which could effectively speed up the inter-processor communication.

Table 2

The GaAs bond-dependent BOP parameters [40]

\begin{tabular}{lclll}
\hline Symbol & Quantity & Ga-Ga & As-As & Ga-As \\
\hline$r_{0}$ & $\begin{array}{c}\text { GSP reference } \\
\text { radius }(\AA)\end{array}$ & 2.4235 & 2.1200 & 2.3800 \\
$r_{c}$ & $\begin{array}{c}\text { GSP characteristic } \\
\text { radius }(\AA)\end{array}$ & 2.4235 & 2.1200 & 2.3800 \\
$m$ & $\begin{array}{c}\text { GSP attractive } \\
\text { exponent }\end{array}$ & 1.4509 & 1.3059 & 1.9652 \\
$n$ & $\begin{array}{c}\text { GSP repulsive } \\
\text { exponent }\end{array}$ & 0.7255 & 0.6529 & 0.9826 \\
$n_{c}$ & $\begin{array}{c}\text { GSP decay } \\
\text { exponent }\end{array}$ & 2.6234 & 2.6304 & 2.6234 \\
$r_{l}$ & $\begin{array}{c}\text { Spline start radius } \\
(\AA)\end{array}$ & 3.0000 & 3.0000 & 3.0000 \\
$r_{\text {cut }}$ & Spline cutoff radius & 3.7000 & 3.6500 & 3.7000 \\
\hline
\end{tabular}




\begin{tabular}{|c|c|c|c|c|}
\hline & $(\AA)$ & & & \\
\hline$\varphi_{0}$ & $\begin{array}{l}\text { Repulsive energy } \\
\text { prefactor }(\mathrm{eV})\end{array}$ & 1.5520 & 3.9800 & 2.1000 \\
\hline$\beta_{\sigma, 0}$ & $\begin{array}{l}\sigma \text { bond integral } \\
\text { prefactor }(\mathrm{eV})\end{array}$ & 1.5233 & 3.0877 & 1.7959 \\
\hline$\beta_{\pi, 0}$ & $\begin{array}{l}\pi \text { bond integral } \\
\text { prefactor }(\mathrm{eV})\end{array}$ & 0.0975 & 0.9263 & 0.3233 \\
\hline$c_{\sigma}$ & $\begin{array}{c}\text { Empirical } \\
\theta_{\sigma} \text { parameter }\end{array}$ & 1.5193 & 3.6016 & 0.8534 \\
\hline$c_{\pi}$ & $\begin{array}{c}\text { Empirical } \\
\theta_{\pi} \text { parameter }\end{array}$ & 1.0000 & 1.0000 & 1.0000 \\
\hline$f_{\sigma}$ & $\begin{array}{l}\text { Band filling } \\
\text { fraction }\left(e^{-} / 8\right)\end{array}$ & 0.4456 & 0.6558 & 0.5000 \\
\hline$k_{\sigma}$ & Skewing prefactor & -25.6485 & 0.7600 & 0.0000 \\
\hline
\end{tabular}

Additionally, the ZBL potential function [42] was employed to describe the interaction of carbon with the GaAs substrate as shown in Eq. (10-11).

$E_{i j}^{Z B L}=\frac{1}{4 \pi \epsilon_{0}} \frac{Z_{i} Z_{j} e^{2}}{r_{i j}} \varphi\left(r_{i j} / a\right)+S\left(r_{i j}\right)$

$\mathrm{a}=\frac{0.46850}{Z_{i}^{0.23}+Z_{j}^{0.23}}$

where $\epsilon_{0}$ refers to the electrical permittivity of vacuum, $e$ means the electron charge, $Z_{i}$ and $Z_{j}$ are the nuclear charges of the two types of atom, $r_{i j}$ is identical to mentioned above, $S\left(r_{i j}\right)$ is a switching function which can limit the energy, force, and curvature down to zero smoothly within cutoff range. Moreover, the inner cutoff and outer cutoff were set to $3.0 \AA$ and $4.0 \AA$, respectively.

\subsection{Parametric analysis}

In this study, Eq. (12) [43]-[45] was employed to compute the temperature of the diamond tip by conversion of average kinetic energy:

$K . E .=\frac{3}{2} N K_{b} T$

where $K . E$. refers to the kinetic energy of the GaAs substrate atoms, $N$ is the number 
of atoms, $K_{b}$ is the Boltzmann constant.

In addition, the virial stress computation method [36] was used to compute the stress components of the GaAs substrate and diamond tip. It can be described as follows [46][48]:

$\sigma_{a b}(i)=\frac{1}{N_{A}} \sum_{i}\left[\frac{m_{i} v_{i}^{a} v_{i}^{b}}{V_{i}}+\frac{1}{2 V_{i}} \sum_{j} F_{i j} \frac{x_{i j}^{a} x_{i j}^{b}}{r_{i j}}\right]$

where $\sigma_{a b}$ refers to six direction symmetric stress components of each atom, $a b$ represents $\mathrm{x}, \mathrm{y}$, and $\mathrm{z}, N_{A}$ is the number of atoms in an area $A, m_{i}$ is the mass of the $i$ atom, $v_{i}^{a}$ and $v_{i}^{b}$ mean the $a$ component and $b$ component of the $i$ atom velocity, $V_{i}$ is the volume of the $i$ atom, $F_{i j}$ is the force between the $i$ atom and the $j$ atom, $x_{i j}^{a}$ and $x_{i j}^{b}$ represent $a$ direction vector and $b$ direction vector of $i$ atom and $j$ atom, $r_{i j}$ is the distance between the $i$ atom and the $j$ atom.

The hydrostatic stress (average of three principal stresses) and von Mises stress of GaAs substrate and diamond tip were further investigated, which could be calculated by Eq. (14) and Eq. (15), respectively [49]-[51]. In this case, the hydrostatic stress and von Mises stress were averaged over a $20 \AA$ box for each GaAs substrate atom.

$$
\begin{aligned}
& \sigma_{\text {hydro }}(i)=\frac{1}{3}\left[\sigma_{1}+\sigma_{2}+\sigma_{3}\right] \\
& \sigma_{v o n}(i)=\left\{\frac { 1 } { 2 } \left[\left(\sigma_{x x}(i)-\sigma_{y y}(i)\right)^{2}+\left(\sigma_{y y}(i)-\sigma_{z z}(i)\right)^{2}+\left(\sigma_{z z}(i)-\sigma_{x x}(i)\right)^{2}+\right.\right. \\
& \left.\left.6\left(\sigma_{x y}^{2}(i)+\sigma_{z y}^{2}(i)+\sigma_{x z}^{2}(i)\right)\right]\right\}^{1 / 2}
\end{aligned}
$$




\section{Results and discussion}

\subsection{Deformation and wear of diamond tip}

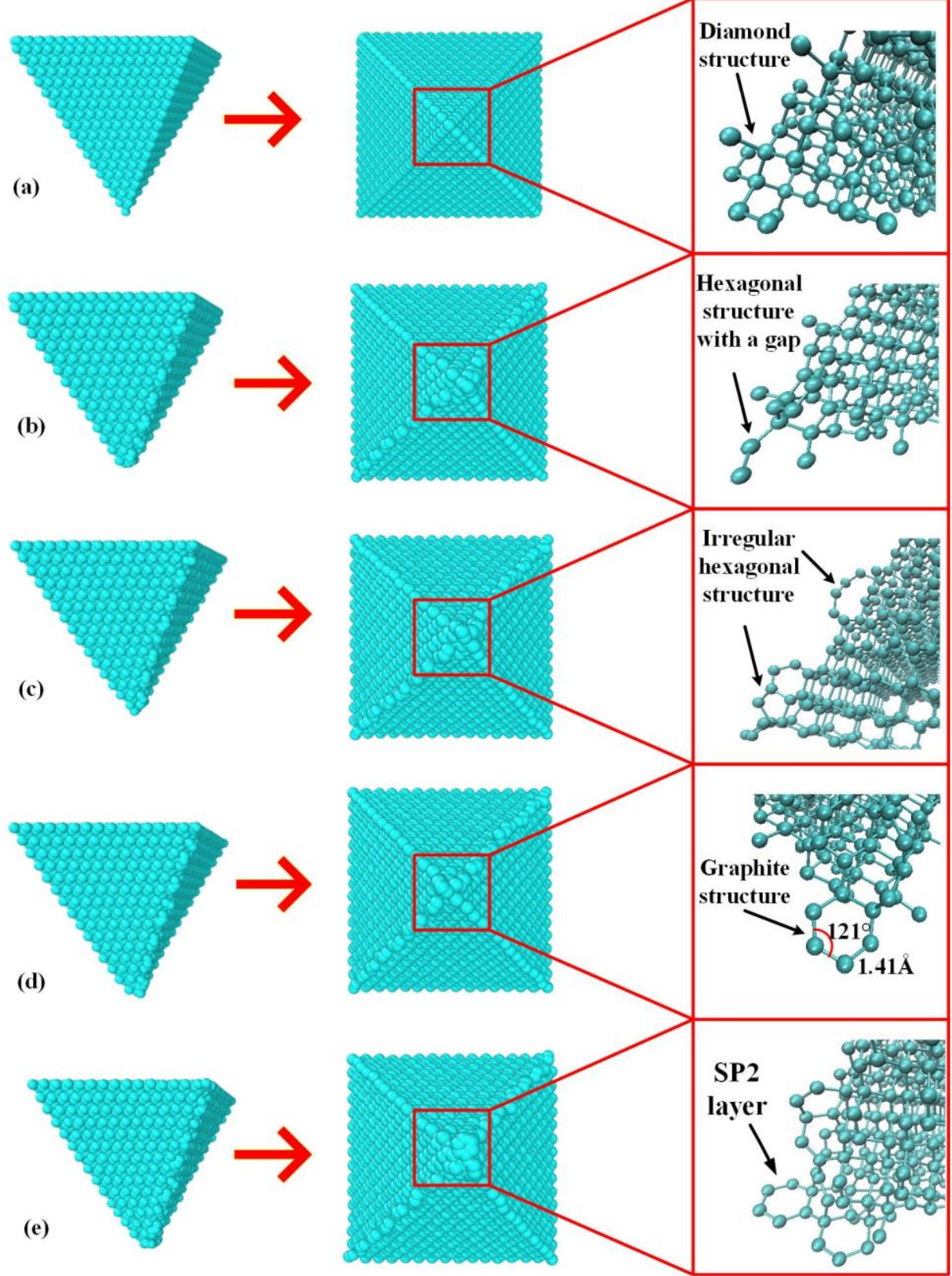

Fig. 2. The observation of structural change of the diamond tip during nanometric cutting of GaAs. (a) initial diamond tip structure. (b-e) the diamond tip structures at the cutting distances of $2 \mathrm{~nm}, 6 \mathrm{~nm}, 10$ $\mathrm{nm}$, and $20 \mathrm{~nm}$, respectively. 


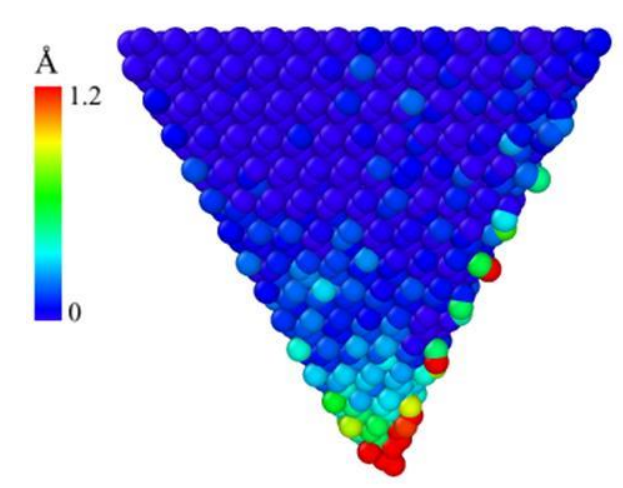

Fig. 3. The atomic displacement of the carbon atoms in the diamond tip after a nanomachining distance of $10 \mathrm{~nm}$

As shown in Fig. 2, the diamond tip experienced elastic-plastic deformation during the nanomachining process caused by a $s p^{3}-s p^{2}$ hybridization transition. The deformation hotspot appeared at the apex of the diamond tip, meanwhile, the cutting edge of the diamond tip showed incipient deformation. Furthermore, from Fig. 3, it can be observed that the maximum atomic displacement of the apex of the diamond tip could reach 0.12 $\mathrm{nm}$. The occurrence of diamond tip elastic-plastic deformation behavior is attributed to high pressure acting on the diamond tip structure [52][53].

Moreover, from the enlarged diagrams of the apex of the diamond tip shown in Fig. 2, it is apparent that a hexagonal structure with two C-C bond gaps (as shown in Fig. 2(b)) forms at the cutting distance of $2 \mathrm{~nm}$. With the nanomachining advancement, an irregular hexagonal structure, as illustrated in Fig. 2(c), was observed at the cutting distance of $6 \mathrm{~nm}$. Then, a complete hexagonal carbon-cycle structure occurred at the distance of $10 \mathrm{~nm}$, as demonstrated in Fig. 2(d). Subsequently, the measured bond angle and bond length of C-C were $121^{\circ}$ and $1.41 \AA$, respectively. This is very close to the graphite hexagonal lattice structure with $121^{\circ} \mathrm{C}-\mathrm{C}$ bond angle and $1.42 \AA \mathrm{C}-\mathrm{C}$ bond length [29][54]. Finally, at the cutting distance of $20 \mathrm{~nm}$, the presence of a clear $s p^{2}$ 
graphite layer structure was observed, as seen in Fig. 2(e). This phenomenon suggests that the apex of the diamond tip transformed from an initial $s p^{3}$ hybridization diamond lattice structure to $s p^{2}$ graphite lattice structure due to the C-C bond transformation and reorganization of bonds. From this, we can infer that the graphitization governs the wear mechanism of diamond tip during AFM tip-based nanomachining of GaAs. Correspondingly, thermal and shear stresses were analyzed in the subsequent sections to investigate the underlying mechanism of graphitization of the diamond tip thoroughly. Further advancement of the machine leads to the graphite layer of diamond to fall off and separated from the diamond tip and create a small concave structure, previously reported by Cheng et al. [55].

\subsection{Structural transformations in GaAs workpiece}

Various stress states in the workpiece were calculated to see if they can induce classical thermodynamic phase transition [47]. As illustrated in Fig. 4, the average hydrostatic stress in the cutting zone experienced a rapid upward trend reaching the maximum scale at $\sim 1.3 \mathrm{GPa}$ and kept a relatively fluctuation between $0.4 \mathrm{GPa}$ and $0.6 \mathrm{GPa}$ during the nanomachining process. Furthermore, the peak hydrostatic stress of about $17.5 \mathrm{GPa}$ occurred in the primary shear slip zone, as shown in Fig. 5. 


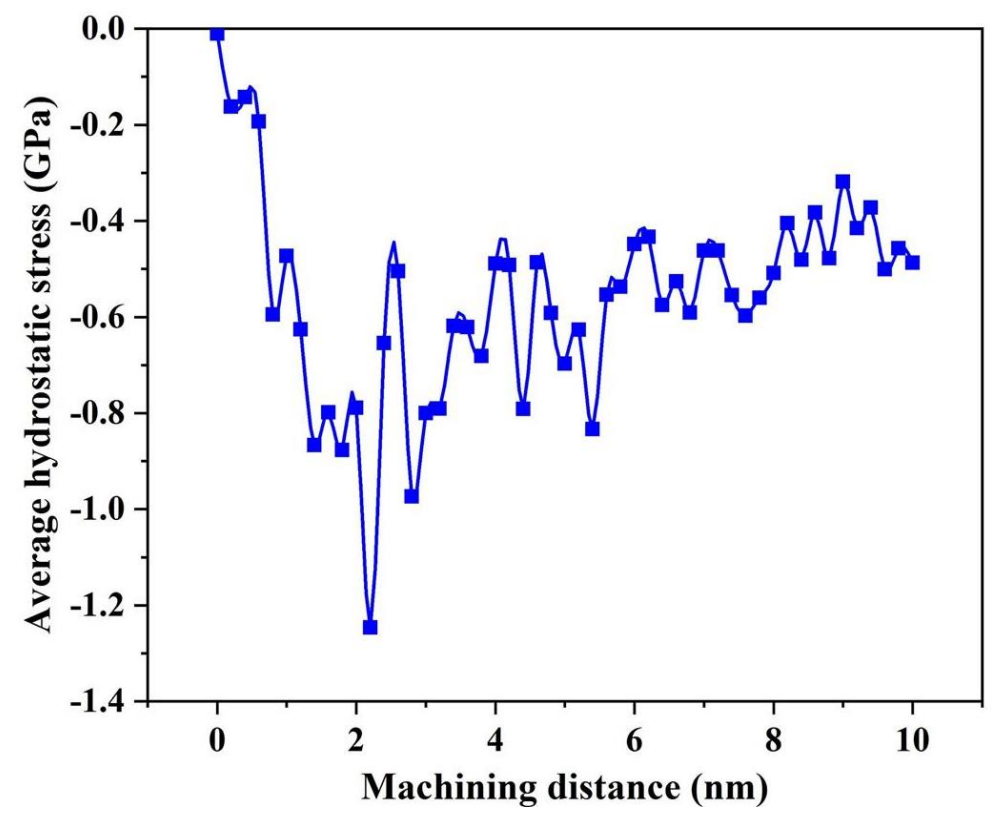

Fig. 4. Variation in the average hydrostatic stress in the cutting zone

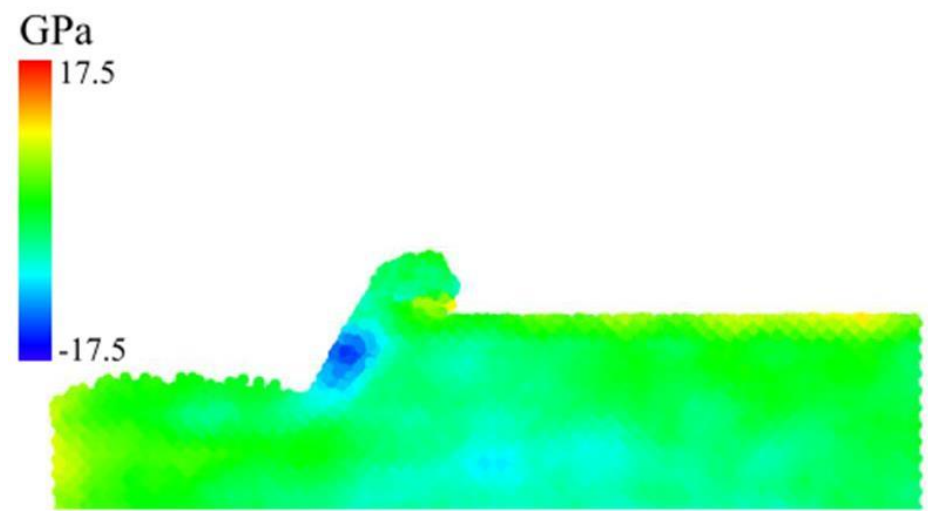

Fig. 5. the distribution of the hydrostatic within the machined single crystal GaAs workpiece.

The peak stress was consistent with the recent experimental reported limits indicating that the single crystal GaAs could well transform from a zinc blende structure (GaAsI) to rocksalt structure (GaAs-II) [56]. To affirm this the coordination number $(\mathrm{CN})$ was computed during the MD simulations, as indicated in Fig. 6. 


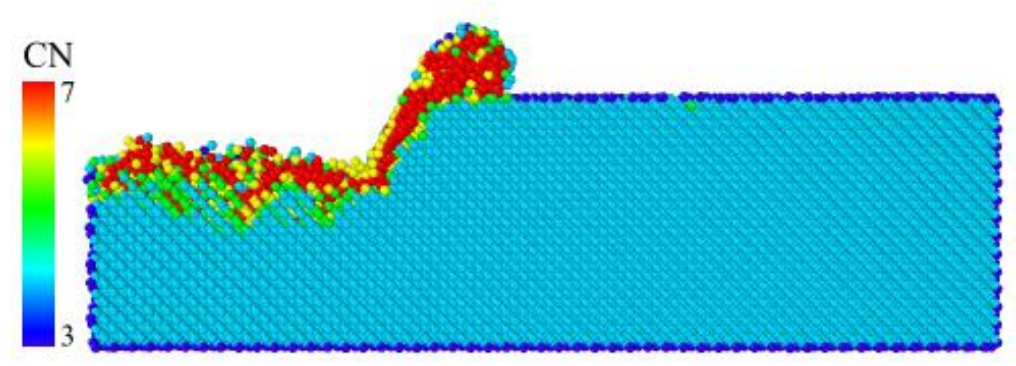

Fig. 6. Variation in the coordination number $(\mathrm{CN})$ during nanomachining distance of $10 \mathrm{~nm}$

The coordination number $(\mathrm{CN})$ ranging from 3 to 7 were observed. A coordination number of 3 represents the dangling bonds on the surface of GaAs, 4 represents the zinc blende structure (GaAs-I), intermediate structures between $\mathrm{CN} \mathrm{4,} \mathrm{CN} 6$ and $\mathrm{CN} 7$ highlights high pressure phases including possible rocksalt structure (GaAs-II), and amorphous structures respectively. It can thus be considered that the occurrence of the high-pressure phase transformation (HPPT) governed the ductility of GaAs during nanometric cutting. More interestingly, a comparison of hydrostatic stress distribution and $\mathrm{CN}$ distribution revealed that a significant amount of rocksalt structures (GaAs-II, shown as yellow color atoms in Fig. 6) were generated at the peak hydrostatic stress area, i.e., primary shear slip zone and the rake face of the diamond tip. Correspondingly, this result signified the evolution of phase transformation of GaAs was in accordance with that of high-pressure during the nanomachining process.

\subsection{Stress state of the diamond tip}

It has been reported by some researchers that the cubic diamond lattice structure can transform to hcp graphite lattice structure under high shear stress state [57][58]. Therefore, the high shear stress is a considerable factor to investigate the underlying 
mechanism for the graphitization of the diamond tip. The evolution of stresses on the diamond tip estimated from the simulations is shown in figure 7.

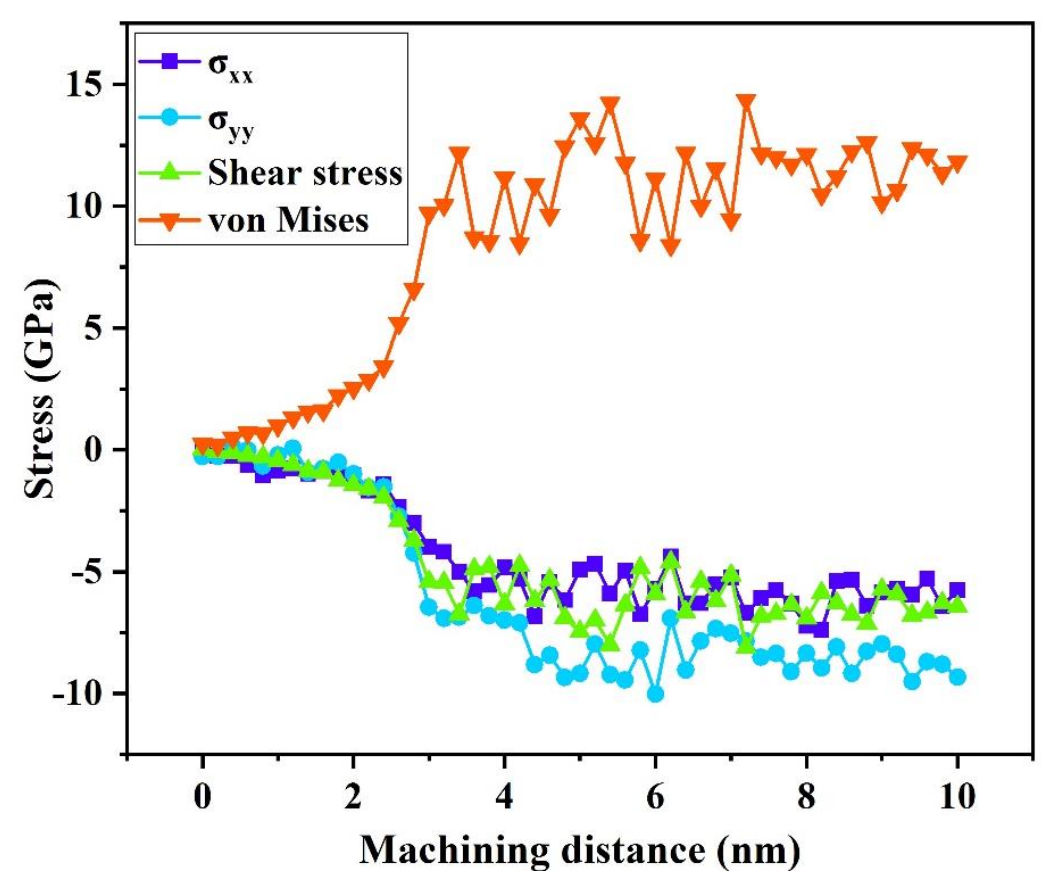

Fig. 7. The stresses variation on the diamond tip

The average shear stress was computed against machining distance, as illustrated in Fig.

7. It was found that the average shear stress fluctuated at a magnitude of $7 \mathrm{GPa}$. In addition, as shown in Fig. 8, after the cutting distance of $10 \mathrm{~nm}$, the peak shear stress was mainly located at the apex of the diamond tip. The magnitude of stress was much lower than the shear stress of $95 \mathrm{GPa}$ that was required to induce the cubic diamond lattice instability to the hcp graphite lattice structure [59]. This indicates that besides shear some other factor also contributes to causing the graphitization of diamond tip during nanometric cutting of single crystal GaAs. 


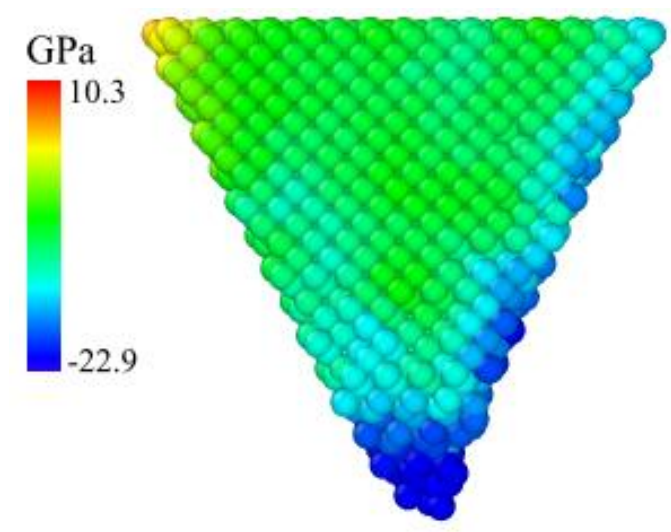

Fig. 8. The distribution of shear stress at the diamond tip after the cutting distance of $10 \mathrm{~nm}$

As earlier shown in figure 7 , the stress components $\sigma_{x x}$ and $\sigma_{y y}$ variation kept fluctuating between $-6 \mathrm{GPa}$ and $-10 \mathrm{GPa}$ indicating the compression state of the diamond tip. The von Mises stress was computed over the simulation period, which is employed to predict the yielding and shape distortion of the GaAs material [60][61]. The average von Mises stress of the diamond tip experienced a relatively stable stage at around $12.5 \mathrm{GPa}$ between the cutting distances $4 \mathrm{~nm}$ and $10 \mathrm{~nm}$.

\subsection{Thermal state of the diamond tip}

Another significant potential factor that may cause graphitization of the diamond tip is the cutting heat. The average temperature of the diamond tip dramatically increased at the start of the nanometric cutting, as illustrated in Fig. 9, and then kept steady at approximately $600 \mathrm{~K}$. The stable average temperature rise of the diamond tip with the increase of cutting distance might be attributed to the quick heat dissipation thanks to the high thermal conductivity of diamond [62]. Another observation from Fig. 9(a) was that the locally highest temperature around $950 \mathrm{~K}$ was concentrated at the apex of the diamond tip, which was also the place of initiation of graphitization. It is known that 
the minimum temperature required to cause the graphitization of diamond is about 940 $\mathrm{K}$ under anaerobic conditions [63], which is basically the same as the high temperature of the apex of the diamond tip based on the simulation results with the same simulation environment. Therefore, it implies that the high temperature at the apex of the diamond tip might weaken the cohesion energy of the C-C bond and further lead to the reorganization of the $\mathrm{C}-\mathrm{C}$ bond with the advancement in the nanometric cutting [55][64]. Correspondingly, cutting heat appears to have triggered the $s p^{3}-s p^{2}$ hybridization transition of the diamond tip.

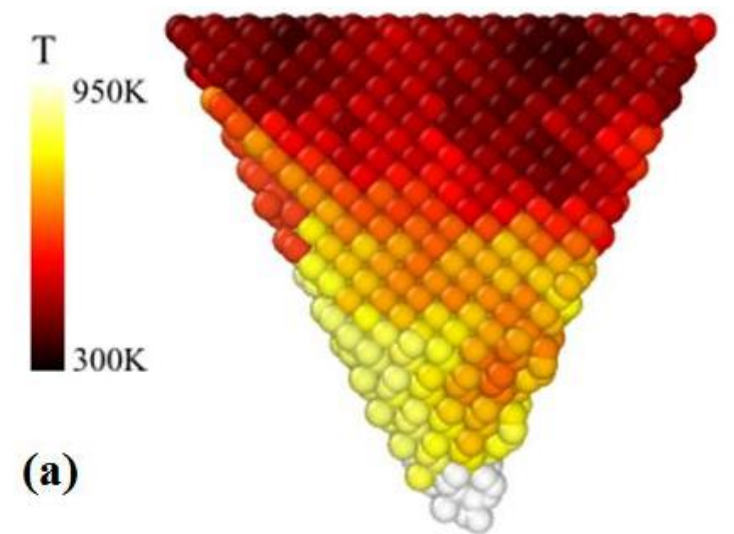




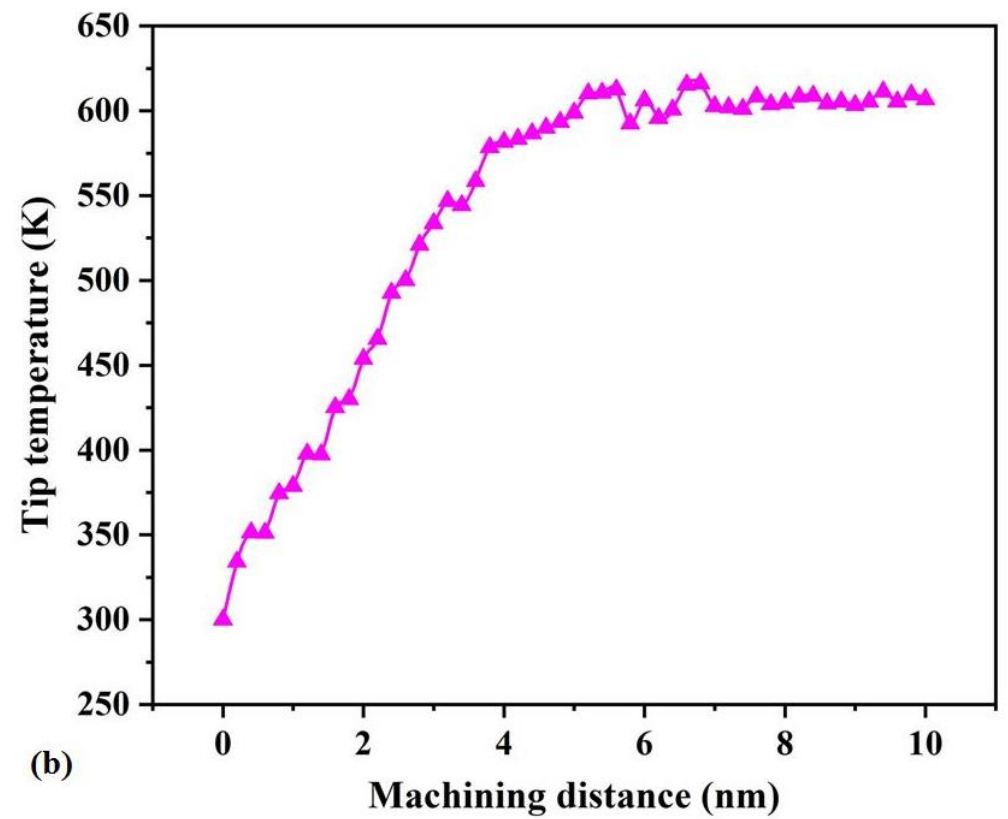

Fig. 9. (a)The temperature distribution of diamond tip at nanomachining distance of $10 \mathrm{~nm}$. (b)The average temperature evolution of diamond tip

\subsection{Graphitization conversion rate of diamond tip}

It is well known that diamond has a coordination number of 4 while graphite has a coordination number of 3 . In this paper, the graphitization conversion rate of the diamond tip was quantified by the division of the number of graphitized atoms to the total atom number (13107 atoms) of the diamond tip. To eliminate the influence of dangling bonds (292 atoms) of the diamond tip surface, the total number of $\mathrm{s}$ graphitization atoms was calculated via the recorded number of $\mathrm{CN} 3$ atoms deducting the initial number of dangling bonds atoms. This is akin to the research methodology that has previously been followed by one of the co-authors of this paper [29].

The graphitization conversion rate of the diamond tip is illustrated in Fig. 10. The trend of the graphitization conversion rate of the diamond tip could be split into two regions 
during the course of nanomachining. The initially growing graphitization region implied that the initial rapid wear up to a cutting distance of $14 \mathrm{~nm}$ was more severe. Later stages of wear were somewhat steady. A similar trend of wear of diamond tool was found during the diamond turning of silicon [65] and silicon carbide [29] although the final rapid tool failure stage and final rapid wear stage did not reach in the MD simulation due to the short cutting distance. Therefore, this curve suggested that the continuous graphitization of diamond tip might lead to tool failure during nanometric cutting of GaAs.

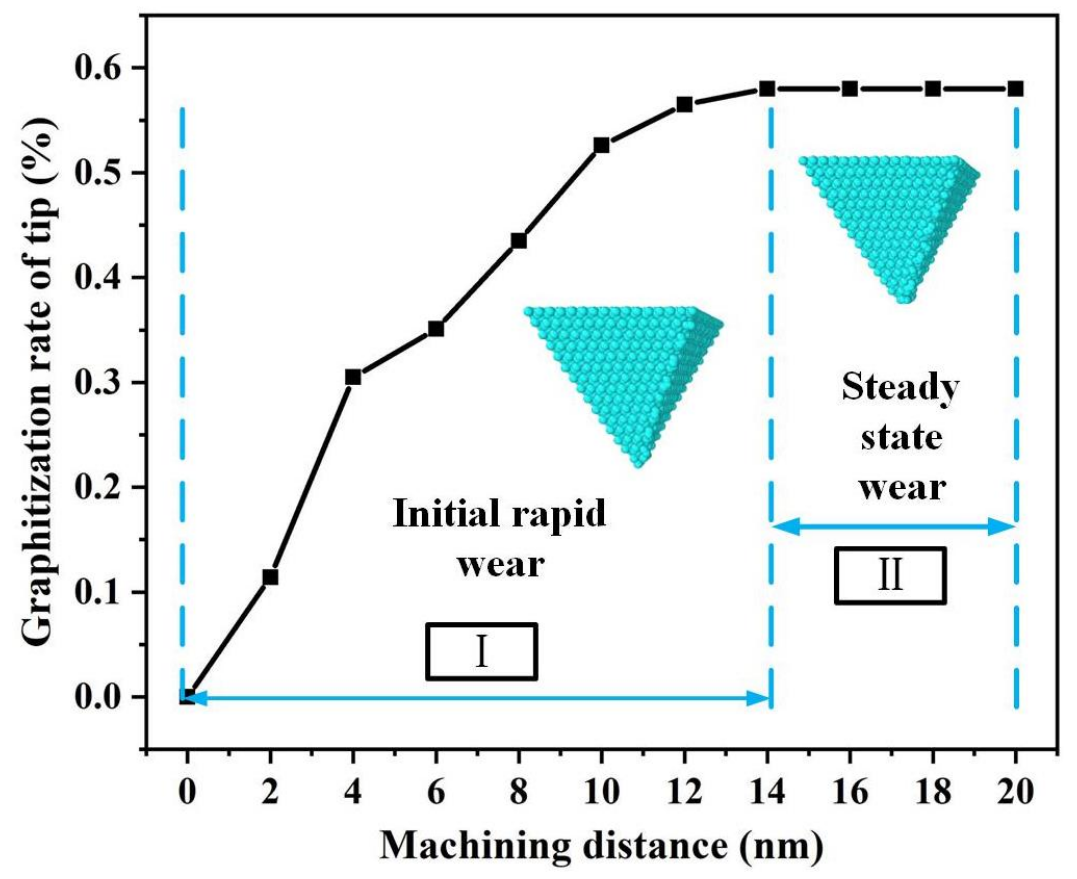

Fig. 10. The graphitization conversion rate of diamond tip

\section{Conclusions}

In this work, the wear mechanism of the diamond tip against GaAs during the AFM tipbased nanomachining process was investigated by using MD simulations. Based on the 
aforementioned discussions, the main conclusions could be as follows:

1. $s p^{3}-s p^{2}$ hybridization transition leading to graphitization of diamond is the dominant wear mechanism of the diamond tip during nanometric cutting of GaAs.

2. The root cause of diamond tip graphitization during nanometric cutting of GaAs was found to be the cutting heat rather than the high shear at the contact interface in the cutting zone.

3. Some indications of high-pressure phase transformation of the GaAs were obtained by using the coordination number method within the simulation and this aspect suggested a high likelihood of the phase transition of GaAs from zinc blende structure (GaAs-I) to rocksalt structure (GaAs-II) during the nanomachining process.

4. The variation in the variation of the graphitization conversion rate of the diamond tip against the cutting distance showed two distinct phases of wear with the initial stage suggesting severe wear followed by steady-state wear.

\section{Acknowledgements}

The authors would like to thank EPSRC (EP/K018345/1, EP/T024844/1) and the Royal Society-NSFC international exchange programme (IEC\NSFC 181474 ) to provide financial support to this research. The authors also acknowledge the use of the EPSRC (EP/K000586/1) funded ARCHIE-WeSt High-Performance Computer at the University of Strathclyde for the MD simulation study. 
SG acknowledges the financial support provided by the UKRI via Grants No.: EP/K503241/1 and EP/L016567/1, EP/S013652/1, EP/T001100/1 and EP/S036180/1, H2020 via Cost Actions CA15102, CA18125, CA18224 and CA16235, Royal Academy of Engineering via Grant No. IAPP18-19\295 (Indo-UK partnership), EXPP2021\1\277, Grant No. TSP1332 (South Africa- UK partnership) and Newton Fellowship award from the Royal Society (NIF\R1\191571).

\section{Data Availability Statement}

All data underpinning this publication are openly available from the University of Strathclyde KnowledgeBase at https://doi.org/10.15129/01270576-2ee6-452c-927ab5a0a89a0921.

Conflict of Interest: The authors declare that they have no conflict of interest.

\section{References}

[1] A. Ghalgaoui, K. Reimann, M. Woerner, T. Elsaesser, C. Flytzanis, and K. Biermann, "Resonant Second-Order Nonlinear Terahertz Response of Gallium Arsenide," Phys. Rev. Lett., vol. 121, no. 26, p. 266602, 2018.

[2] X. C. Zhang, Y. Jin, K. Yang, and L. J. Schowalter, "Resonant nonlinear susceptibility near the GaAs band gap," Phys. Rev. Lett., vol. 69, no. 15, pp. 2303-2306, 1992.

[3] L. Pantoli et al., "Gallium arsenide $0.5-18 \mathrm{GHz}$ antenna frontend with integrated limiter and differential to single ended low-noise amplifier," IET 
Microwaves, Antennas Propag., vol. 12, no. 6, pp. 947-953, 2018.

[4] Ş. Ţălu, P. Nikola, D. Sobola, A. Achour, and S. Solaymani,

"Micromorphology investigation of GaAs solar cells: case study on statistical surface roughness parameters," Journal of Materials Science: Materials in Electronics, vol. 28, no. 20. pp. 15370-15379, 2017.

[5] J. Yoon et al., "GaAs photovoltaics and optoelectronics using releasable multilayer epitaxial assemblies," Nature, vol. 465, no. 7296, pp. 329-333, 2010.

[6] A. W. Bett, F. Dimroth, G. Stollwerck, and O. V. Sulima, "III-V compounds for solar cell applications," Appl. Phys. A Mater. Sci. Process., vol. 69, no. 2, pp. 119-129, 1999.

[7] L. Hu, Y. Wang, and M. Xu, "Experimental study of recovery time of a bulk gallium arsenide avalanche semiconductor switch in low-energy-triggered mode," Electron. Lett., vol. 55, no. 12, pp. 711-712, 2019.

[8] J. Chen, X. Luo, F. Ding, X. Rao, and J. Zhang, "Fundamental study of diamond turning of single crystal gallium arsenide," Precis. Eng., vol. 62, pp. 71-82, 2020.

[9] Z. J. Y. F.Z. Fang, "Ultra-precision cutting for gallium arsenide," in In Proc. ASPE Annual Meeting, 1999, pp. 62-66.

[10] M. Versen, B. Klehn, U. Kunze, D. Reuter, and A. D. Wieck, "Nanoscale devices fabricated by direct machining of GaAs with an atomic force microscope," Ultramicroscopy, vol. 82, no. 1-4, pp. 159-163, 2000. 
[11] Y. Yan, Y. Geng, and Z. Hu, "Recent advances in AFM tip-based nanomechanical machining," Int. J. Mach. Tools Manuf., vol. 99, pp. 1-18, 2015.

[12] C. K. Hyon, S. C. Choi, S. W. Hwang, D. Ahn, Y. Kim, and E. K. Kim, "Direct nanometer-scale patterning by the cantilever oscillation of an atomic force microscope," Appl. Phys. Lett., vol. 75, no. 2, pp. 292-294, 1999.

[13] H. W. Schumacher, U. F. Keyser, U. Zeitler, R. J. Haug, and K. Eberl, "Controlled mechanical AFM machining of two-dimensional electron systems: Fabrication of a single-electron transistor," Phys. E Low-Dimensional Syst. Nanostructures, vol. 6, no. 1, pp. 860-863, 2000.

[14] F. Zhang, B. Meng, Y. Geng, Y. Zhang, and Z. Li, "Friction behavior in nanoscratching of reaction bonded silicon carbide ceramic with Berkovich and sphere indenters," Tribol. Int., vol. 97, pp. 21-30, 2016.

[15] Y. Geng, Y. Yan, Y. He, and Z. Hu, "Investigation on friction behavior and processing depth prediction of polymer in nanoscale using AFM probe-based nanoscratching method," Tribol. Int., vol. 114, no. April, pp. 33-41, 2017.

[16] Y. Geng, Y. Yan, Y. Zhuang, and Z. Hu, "Effects of AFM tip-based direct and vibration assisted scratching methods on nanogrooves fabrication on a polymer resist,” Appl. Surf. Sci., vol. 356, pp. 348-354, 2015.

[17] F. Zhang, B. Meng, Y. Geng, and Y. Zhang, "Study on the machined depth when nanoscratching on $6 \mathrm{H}-\mathrm{SiC}$ using Berkovich indenter: Modelling and experimental study," Appl. Surf. Sci., vol. 368, pp. 449-455, 2016. 
[18] Y. Yan, Y. Geng, Z. Hu, X. Zhao, B. Yu, and Q. Zhang, "Fabrication of nanochannels with ladder nanostructure at the bottom using AFM nanoscratching method," Nanoscale Res. Lett., vol. 9, no. 1, pp. 1-13, 2014.

[19] Y. Ma, G. Ballesteros, J. M. Zajac, J. Sun, and B. D. Gerardot, "Highly directional emission from a quantum emitter embedded in a hemispherical cavity," Opt. Lett., vol. 40, no. 10, p. 2373, 2015.

[20] A. Mir, X. Luo, and J. Sun, "The investigation of influence of tool wear on ductile to brittle transition in single point diamond turning of silicon," Wear, vol. 364-365, pp. 233-243, 2016.

[21] S. Saketi, U. Bexell, J. Östby, and M. Olsson, "On the diffusion wear of cemented carbides in the turning of AISI 316L stainless steel," Wear, vol. 430431, no. March, pp. 202-213, 2019.

[22] B. M. Lane, T. A. Dow, and R. Scattergood, "Thermo-chemical wear model and worn tool shapes for single-crystal diamond tools cutting steel," Wear, vol. 300, no. 1-2, pp. 216-224, 2013.

[23] M. Binder, F. Klocke, and B. Doebbeler, “Abrasive wear behavior under metal cutting conditions," Wear, vol. 376-377, pp. 165-171, 2017.

[24] L. Zou, J. Yin, Y. Huang, and M. Zhou, "Essential causes for tool wear of single crystal diamond in ultra-precision cutting of ferrous metals," Diam. Relat. Mater., vol. 86, no. 174, pp. 29-40, 2018.

[25] G. Li, M. Zula, W. Pan, C. Wen, and S. Ding, "The manufacturing and the application of polycrystalline diamond tools - A comprehensive review," J. 
Manuf. Process., vol. 56, pp. 400-416, 2020.

[26] S. Goel et al., "Horizons of accelerated molecular dynamics in digital manufacturing of new generation advanced materials," Mater. Today Chem., 2020.

[27] F. Xie, Z. J. Jin, X. G. Guo, and S. J. Shi, "Wear Mechanism of Diamond Tool against Mold Steel in Single Point Diamond by Molecular Dynamics," Appl. Mech. Mater., vol. 851, pp. 186-190, 2016.

[28] S. Goel, X. Luo, and R. L. Reuben, "Wear mechanism of diamond tools against single crystal silicon in single point diamond turning process," Tribol. Int., vol. 57, pp. 272-281, 2013.

[29] S. Goel, X. Luo, and R. L. Reuben, "Molecular dynamics simulation model for the quantitative assessment of tool wear during single point diamond turning of cubic silicon carbide," Comput. Mater. Sci., vol. 51, no. 1, pp. 402-408, 2012.

[30] G. Dong, X. Wang, and S. Gao, "Molecular dynamics simulation and experiment research of cutting-tool wear mechanism for cutting aluminum alloy," Int. J. Adv. Manuf. Technol., vol. 96, no. 1-4, pp. 1123-1137, 2018.

[31] S. J. Plimpton, "Fast parallel algorithms for short range molecular dynamics," J. Comput. Phys., vol. 117, pp. 1-19, 1995.

[32] S. Nose, "A unified formulation of the constant temperature moleculardynamics methods," J. Chem. Phys., vol. 81, pp. 511-519, 1984.

[33] D. T. Infield et al., "Main-chain mutagenesis reveals intrahelical coupling in an ion channel voltage-sensor," Nat. Commun., vol. 9, no. 1, pp. 1-10, 2018. 
[34] A. Stukowski, "Visualization and analysis of atomistic simulation data with OVITO-the Open Visualization Tool," Model. Simul. Mater. Sci. Eng., vol. 18, no. $1,2010$.

[35] J. Tersoff, "Modeling solid-state chemistry: Interatomic potentials for multicomponent systems," Phys. Rev. B, vol. 39, no. 8, pp. 5566-5568, 1989.

[36] S. Goel, X. Luo, A. Agrawal, and R. L. Reuben, "Diamond machining of silicon: A review of advances in molecular dynamics simulation," Int. J. Mach. Tools Manuf., vol. 88, pp. 131-164, 2015.

[37] H. Dai, S. Li, and G. Chen, "Molecular dynamics simulation of subsurface damage mechanism during nanoscratching of single crystal silicon," Proc. Inst. Mech. Eng. Part J J. Eng. Tribol., vol. 0, no. 0, pp. 1-13, 2018.

[38] D. K. Ward, X. W. Zhou, B. M. Wong, F. P. Doty, and J. A. Zimmerman, "Analytical bond-order potential for the cadmium telluride binary system," Phys. Rev. B - Condens. Matter Mater. Phys., vol. 85, no. 11, pp. 1-19, 2012.

[39] D. G. Pettifor and I. I. Oleinik, "Analytic bond-order potential for open and close-packed phases," Phys. Rev. B - Condens. Matter Mater. Phys., vol. 65, no. 17 , pp. 1-4, 2002.

[40] D. A. Murdick, X. W. Zhou, H. N. G. Wadley, D. Nguyen-Manh, R. Drautz, and D. G. Pettifor, "Analytic bond-order potential for the gallium arsenide system,” Phys. Rev. B - Condens. Matter Mater. Phys., vol. 73, no. 4, pp. 1-20, 2006.

[41] L. Goodwin, C. M. Goringe, D. R. Bowler, A. P. Sutton, and M. W. Finnis, 
"Generating Transferable Tight-Binding Parameters: Application to Silicon," Europhys. Lett., vol. 9, no. 7, pp. 701-706, 1989.

[42] J. F. Ziegler, M. D. Ziegler, and J. P. Biersack, "SRIM - The stopping and range of ions in matter (2010)," Nucl. Instruments Methods Phys. Res. Sect. B Beam Interact. with Mater. Atoms, vol. 268, no. 11-12, pp. 1818-1823, 2010.

[43] J. Wang, X. Zhang, and F. Fang, "Molecular dynamics study on nanometric cutting of ion implanted silicon," Comput. Mater. Sci., vol. 117, pp. 240-250, 2016.

[44] S. Goel, W. Bin Rashid, X. Luo, A. Agrawal, and V. K. Jain, “A Theoretical Assessment of Surface Defect Machining and Hot Machining of Nanocrystalline Silicon Carbide,” J. Manuf. Sci. Eng., vol. 136, no. 2, p. 021015, 2014.

[45] P. A. Romero, G. Anciaux, A. Molinari, and J. F. Molinari, "Insights into the thermo-mechanics of orthogonal nanometric machining," Comput. Mater. Sci., vol. 72, pp. 116-126, 2013.

[46] X. Q. B. Liu, "How to compute the atomic stress objectively?," J. Comput. Theor. Nanosci., vol. 6, no. 5, pp. 1081-1089, 2009.

[47] H. Dai, G. Chen, C. Zhou, Q. Fang, and X. Fei, “A numerical study of ultraprecision machining of monocrystalline silicon with laser nano-structured diamond tools by atomistic simulation," Appl. Surf. Sci., vol. 393, pp. 405-416, 2017.

[48] Y. S. Noriyuki Miyazaki, "Calculation of Mechanical Properties of Solids 
Using Molecular Dynamics Method," JSME Int. journal. Ser. A, Mech. Mater. Eng., vol. 39, no. 4, pp. 606-612, 1996.

[49] F. Wakai, Y. Shinoda, and T. Akatsu, "Tensor-virial equation for deformation of a particle in viscous sintering," J. Am. Ceram. Soc., vol. 95, no. 9, pp. 27852787, 2012.

[50] T. W. Lion and R. J. Allen, "Computing the local pressure in molecular dynamics simulations," J. Phys. Condens. Matter, vol. 24, no. 28, pp. 1-6, 2012.

[51] S. Goel, A. Kovalchenko, A. Stukowski, and G. Cross, "Influence of microstructure on the cutting behaviour of silicon," Acta Mater., vol. 105, pp. 464-478, 2016.

[52] N. Adachi, N. Wu, Y. Todaka, H. Sato, and R. Ueji, "Phase transformation in $\mathrm{Fe}-\mathrm{Mn}-\mathrm{C}$ alloys by severe plastic deformation under high pressure," Mater. Lett., vol. 185, pp. 109-111, 2016.

[53] J. Gasc et al., "High-pressure, high-temperature plastic deformation of sintered diamonds," Diam. Relat. Mater., vol. 59, pp. 95-103, 2015.

[54] A. Silverman, J. Adler, and R. Kalish, "Diamond membrane surface after ionimplantation-induced graphitization for graphite removal: Molecular dynamics simulation," Phys. Rev. B - Condens. Matter Mater. Phys., vol. 83, no. 22, pp. $1-9,2011$.

[55] K. Cheng, X. Luo, R. Ward, and R. Holt, "Modeling and simulation of the tool wear in nanometric cutting," Wear, vol. 255, no. 7-12, pp. 1427-1432, 2003. 
[56] S. Ono and T. Kikegawa, "Phase transformation of GaAs at high pressures and temperatures," J. Phys. Chem. Solids, vol. 113, pp. 1-4, 2018.

[57] Y. G. Gogotsi, A. Kailer, and K. G. Nickel, "Transformation of diamond to graphite," Nature, vol. 401, no. 6754, pp. 663-664, 1999.

[58] M. Popov, "Stress-induced phase transitions in diamond," High Press. Res., vol. 30, no. 4, pp. 670-678, 2010.

[59] H. Chacham and L. Kleinman, "Instabilities in diamond under high shear stress,” Phys. Rev. Lett., vol. 85, no. 23, pp. 4904-4907, 2000.

[60] A. D. Freed, "Hencky strain and logarithmic rates in Lagrangian analysis," Int. J. Eng. Sci., vol. 81, pp. 135-145, 2014.

[61] G. L. W. Cross, "Silicon nanoparticles: Isolation leads to change," Nat. Nanotechnol., vol. 6, no. 8, pp. 467-468, 2011.

[62] S. Goel, X. Luo, R. L. Reuben, and H. Pen, "Influence of temperature and crystal orientation on tool wear during single point diamond turning of silicon," Wear, vol. 284-285, pp. 65-72, 2012.

[63] N. S. Xu, J. Chen, and S. Z. Deng, "Effect of heat treatment on the properties of nano-diamond under oxygen and argon ambient," Diam. Relat. Mater., vol. 11, no. 2, pp. 249-256, 2002.

[64] K. Maekawa and A. Itoh, "Friction and tool wear in nano-scale machining-a molecular dynamics approach," Wear, vol. 188, pp. 115-122, 1995.

[65] I. Durazo-Cardenas, P. Shore, X. Luo, T. Jacklin, S. A. Impey, and A. Cox, “3D characterisation of tool wear whilst diamond turning silicon," Wear, vol. 
262, no. 3-4, pp. 340-349, 2007. 